\title{
Using Kernel Estimator and K-Mean Clustering Approach to Hand Gesture Recognition
}

\section{Ban A. Mitras}

dr.banah.mitras@gmail.com

College of Computer Sciences and Mathematics

University of Mosul, Mosul, Iraq

Received on: 26/09/2012

Accepted on: 30/01/2013

\begin{abstract}
In this paper we used non parametric density estimator (Kernel Estimator) to estimate probability density function for Image data of Hand Gesture and warping Hand Gesture and we see the curve for Kernel Estimator and combine the curve between Kernel Estimator and normal Distribution. Programs written using the language Matlab (R2009a) .
\end{abstract}

Keyword: Hand Gesture, Kernel estimator, Pattern Recognition.

$$
\begin{aligned}
& \text { استخدام المقدّر أللبي وأسلوب العنقدة بمعدل - للتعرف على إيماءة اليد } \\
& \text { بان احمد متراس آلاء محمود محمد } \\
& \text { كلية علوم الحاسوب والرياضيات } \\
& \text { جامعة الدوصل، الموصل، العرق }
\end{aligned}
$$

$$
\begin{aligned}
& \text { تاريخ قبول البحث: 2013/01/30 } \\
& \text { تاريخ استلام البحث: 2012/09/26 }
\end{aligned}
$$

\section{الملخص}

تم في هذا البحث استخدام مقدر الكثافة اللامعلمية (المقدر أللبي Kernel Estimator) لتقدير دالة كثافة الاحتمال لبيانات صورة إيماءة اليد الاعتيادية والمشوهة، وقد تبين أن مقدر الكثافة أللبي قادر على تقدير دالة

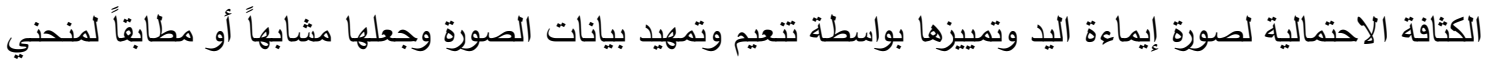

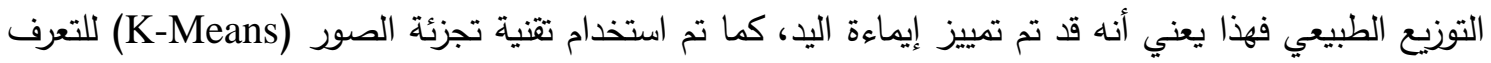

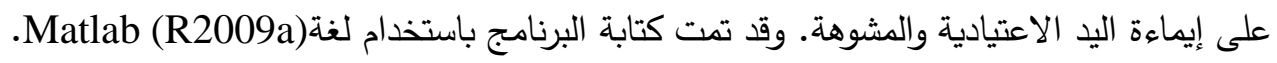
الكلمات المفتاحية: ايماءة اليد، المقدر اللبي، تمييز الأنماط.

:Introduction مقدمة

أن تعقد الظواهر وتثعبها وتطور العلاقات وعدم توافر معلومات كاملة عنها كأن تكون الظاهرة تحدث لأول مرة أو كون النماذج المفترضة لا تحتوي على خصائص يمكن التعويل عليها، كل هذه الأسباب جعلت

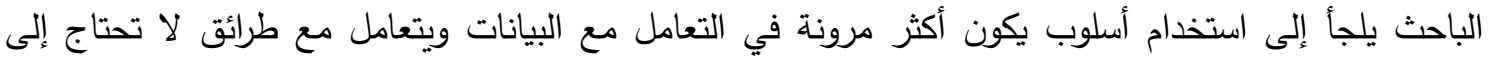
فرضيات أو في أقل تقدير تحتاج إلى فرضيات أقل لاسيما فيما يتعلق بعدم الحصول على معلمات أو أن بيانات 
العينة تكون رتبوية أو نسبوية أو وصفية، وهي موجودة في الكثير من حالات التطبيق ويطلق على هذا الأسلوب

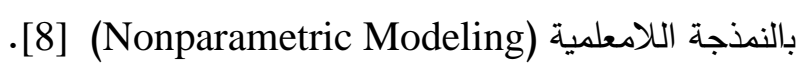

من الطرائق المهمة في التمييز هي تمييز الحركات البشرية بوساطة الإيماءة لما لها من أهمية خاصة في لغة الصم والبكم، ويتم أخذها بوصفها إثارة مدخلة. على سبيل المثال نظام تمييز الإيماءة يتم بوساطة متسلسلة

$$
\text { لعدد من الأوقات لحركة اليد [10]. }
$$

إن الافتراض الأساس للأنموذج الإحصائي يتمثل في إمكانية تمييز الإشارة وبصورة جيدة بوصفها عملية

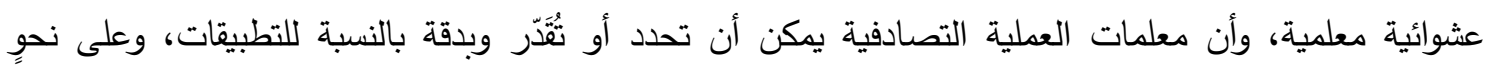
خاص في عملية تمييز الإيماءة، فإنه يمكن تطبيق نماذج الإشارة التصادفية ونماذج الإشارة المحددة وبنجاح.

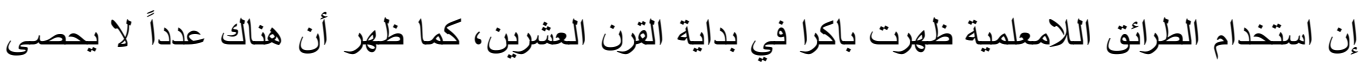

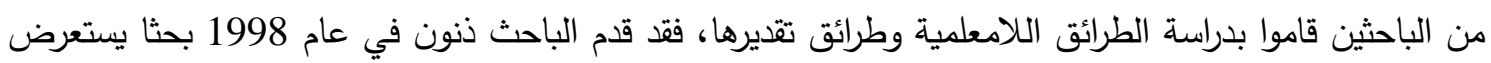
فيه التقدير اللبي بوصفه أسلوبا بيانيا حديثا [2]. وقد قام الباحث حمو في 2000 بمقارنة ثلاثة مقدرات لدالة الانحدار اللامعلمية مقترحا دالة لبية kernel

هو أسلوب بياني لامعلمي، يمكن استخدامه لتقدير أي دالة إحصائية، أما خصائصه فهي البساطة

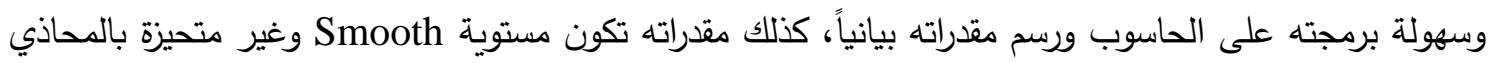

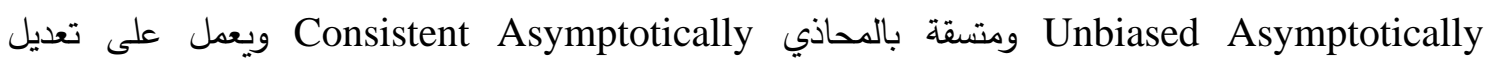
المشاهدات وعلى تقدير دالة الانحدار اللامعلمي التقديرية إلى دالة الانحدار اللامعلمي الحقيقية.[1] بادئ إن مقدر الكثافة أللبي (Kernel) والذي تم اقتراحه من قبل الباحثين Rosenblatt معيل (1956) و و(1962) Parzen المتغير ويراد عرضها بيانيا، فإن المقدر أللبي Kernel سوف يزودنا بوسيلة فعالة لتحقيق هذا الهدف وإيجاد

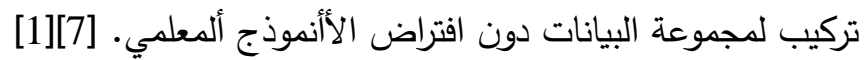
لنفرض أن احتمال مجهولة f(x) • إن المقدر أللبي لهذه الدالة هو: [2] $\hat{f}_{x}(x)=\frac{1}{n h} \sum_{i=1}^{n} K\left(\frac{x-X_{i}}{h}\right)$

إذ إن دالة لُبية (أو دالة النافذة Window Function) وهي عبارة عن دالة رياضية حقيقية محددة تقوم بتسوية المقدر وإزالة التعرجات منه (أي تتقيته، ولهذا سميت نافذة) وتحقق الثرطين الآتيين:

I $K(x) \geq 0 \quad$ for all $x \geq 0$.

II $\int_{-\infty}^{\infty} K(x) d x=1$.

أي إن الدالة اللبية يجب أن تحقق شرطي دالة كثافة الاحتمال كليهما [1]. 


\section{Function Kernel Selection اختيار الدوال اللبية}

تستعمل الدوال اللبية Kernel في تقدير كل من دوال الكثافة الاحتمالية، دوال الانحدار ودوال الطيف Kernel يمكن تمييزهما وهما الدوال اللبية ذات اقل تباين Optimal والتي تعمل على تقليل التباين المحاذي والدوال اللبية المثلى (Minimum Variance Kernel) Mean Integrated Square Error (MISE) والتي تعمل على تقليل متوسط مربع الخطأ التكاملي تلئيل

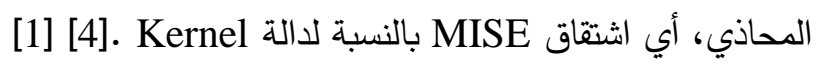

وإن الدالة اللبية Kernel تكون حقيقية ومتماثلة ومحددة ومستمرة، وتكاملها مساو إلى الواحد [9][7].

: Bandwidth 2.2

المعلمة التمهيدية هي h والتي تدعى bandwidth أي عرض الحزمة، معلمة حجم نافذة الانتثار أو

التركيز أو معلمة التباين.

تؤثر المعلمة التمهيدية تأثيرا كبيرا في تمهيد المنحني المقدر واقترابه من المنحنى الحقيقي، إذ بزيادة هذه

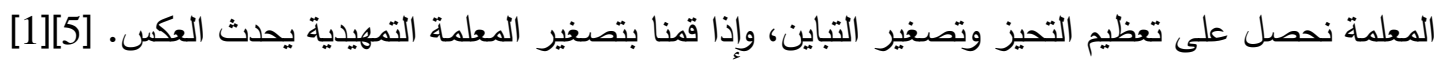

2.3 التمييز باستخدام مقدر الكثافة اللامعلية المقدر أللبي (Kernel Estimator):

يتم التمييز باستخدام مقدر الكثافة اللامعلمية (المقدر أللبي Kernel Estimator) وذلك بملاحظة المنحني الناتج من دالة المقدر أللبي، فإذا كان قريباً أو مشابهاً إلى منحني التوزيع الطبيعي فهذا يعني أن المقدر

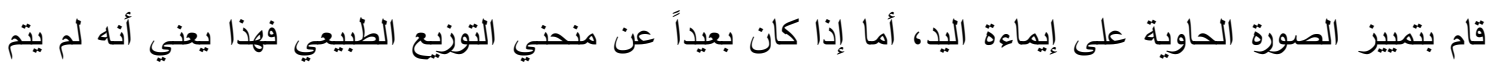

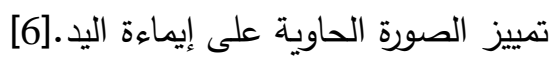

: k-Means Technique -

هي إحدى تقنيات العنقدة المكتثفة في سنة 1967 من قبل العالم MacQueen وعدّها تقنية تصنيفات غير مرشدة وتعتمد على معيار اقل مسافة، إذ تعطى مجموعة من القيم ومحاولة تجزأتها إلى K من العناقيد،

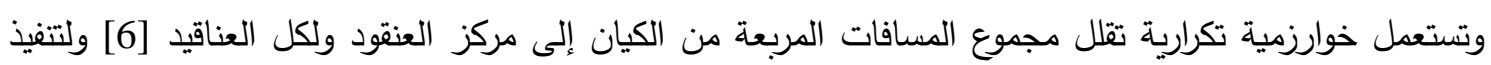
هذه التقنية نطبق الخوارزمية الآتية:

الخطوة الأولى: اختيار مراكز العناقيد عشوائيا الخطوة الثانية: تتسيب كل نقطة X إلى واحد من العناقيد القريبة لها حسب المسافة بين النقطة ومركز العنقود وعلى أساس المسافة الإقليدية وباستعمال العلاقة الآتية:

$\mathrm{X} \in \mathrm{C}_{\mathrm{j}}(\mathrm{t}) \quad$ if $\mathrm{d}\left(\left(\mathrm{X}, \mathrm{M}_{\mathrm{j}}(\mathrm{t})\right)<\mathrm{d}\left(\mathrm{X}, \mathrm{M}_{\mathrm{i}}(\mathrm{t})\right)\right.$

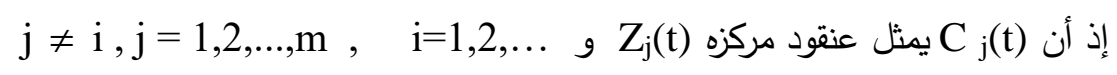

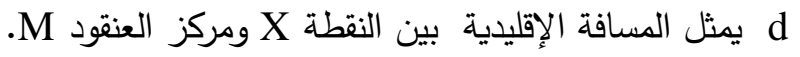

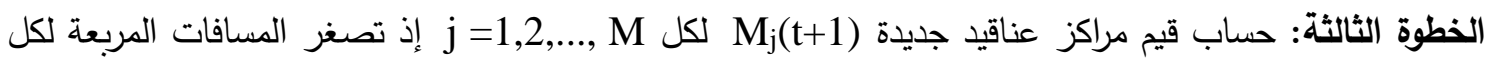
المتجهات في العنقود C Cبالنسبة لمركز العنقود الجديد وعليه يمكن حساب مركز العنقود الجديد

حسب المعادلة الآتية: 
$M_{j}(t+1)=\frac{1}{S_{j}} \sum_{X \in C_{j}(t)} X$

إذ أن:

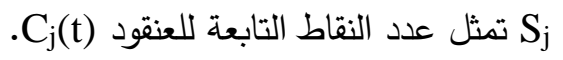

. Cمثل نقطة تابعة للعنقود

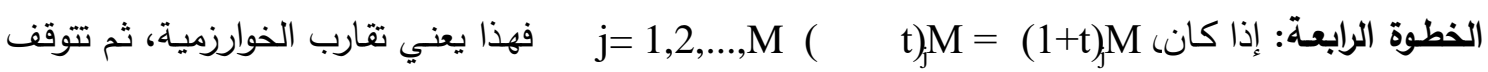

وبخلافه يتم الذهاب إلى الخطوة الثانية.

وهذه الخوارزمية تتغير تبعا للاتي: 1. عدد العناقيد. 2. اختيار القيم البدائية. 3. خصائص البيانات.

5. خوارزمية تجزئة وتقدير دالة كثافة الاحتمال لصورة إيماءة اليد الاعتيادية

Segmentation and Estimator Function Algorithm for Hand Gesture Image

$$
\text { فيما يأتي خطوات الخوارزمية المقترحة: }
$$

الخطوة الأولى: قراءة المقطع الفيديو الذي يحتوي على الإيماءة، ثم أخذ إطار منه على شكل صورة وقراءتها.

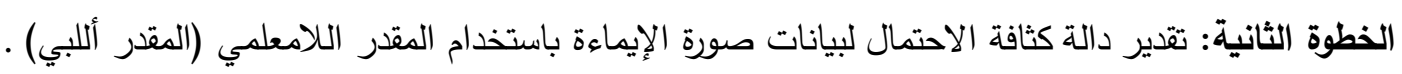

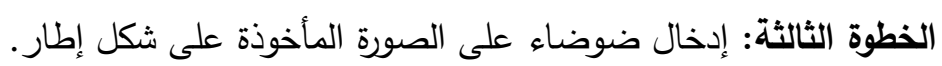

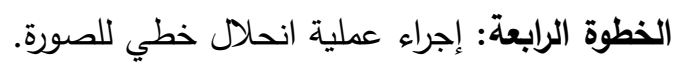

الخطوة الخامسة: حساب قانون كاوسيان الخطي (قانون الانحلال) . Y = AX + إذ أن A تمثل مصفوفة القيم الميزة وn نسبة الضوضاء المضافة للصورة.

الخطوة السادسة: إزالة الضوضاء من الصورة باستخدام المرشح الأوسطي. الخطوة السابعة: أخذ تحدد للحافات باستخدام محدد كاني لإظهار حدود الصورة وحافاتهاتها. الخطوة الثامنة: إجراء ترشيح للصورة مع المرشح المكون وخزنه في مصفوفة مثل J باستخدام أحد المرشحات canny; Sobel ; log; Laplace,...

الخطوة التاسعة: إضافة كثافة للصورة المرشحة وخزنها في مصفوفة مثل K

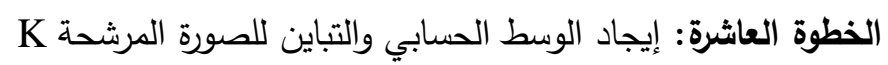

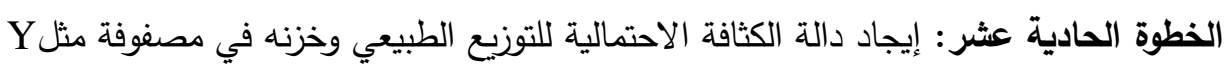
الخطوة الثانية عشر : تجزئة صورة إيماءة اليد باستخدام تقنية K-Means والتعرف عليها. الخطوة الثالثة عشر : عرض الصور كما في الثكل المرقم (1). 
original image

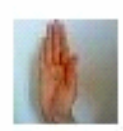

A

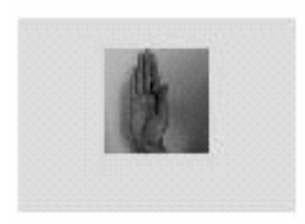

canny filter

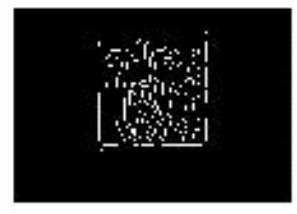

Ksdensity image

0.05

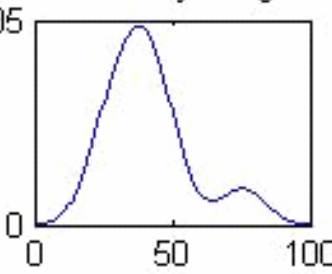

Y

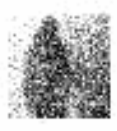

k-means

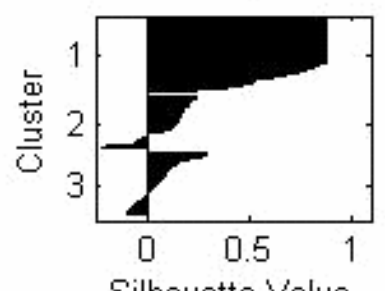

noise image

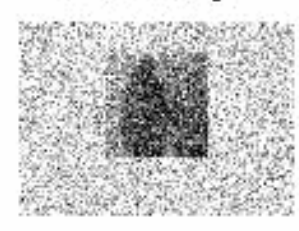

filter noise image

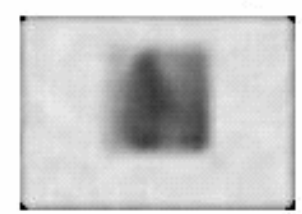

intensity image

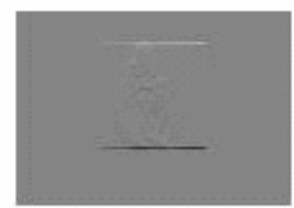

Silhouette Value

الثكل (1). يمثل الصورة الاعتيادية الأولى التي طبقت عليها الخوارزمية اللامعمية لتقدير دالة كثافة الاحتمالية لصورة إيماءة اليد الاعتيادية

جدول (1). يمثل مراحل أسلوب العنقدة بمعدل k-mean) k) لصورة إيماءة اليد الاعتيادية في الثكل (1)

\begin{tabular}{|c|c|c|c|}
\hline iter & phase & num & sum \\
\hline 1 & 1 & 210 & $2.13983 e+008$ \\
\hline 2 & 1 & 36 & $2.04656 e+008$ \\
\hline 3 & 1 & 22 & $2.01228 e+008$ \\
\hline 4 & 1 & 6 & $2.00596 e+008$ \\
\hline 5 & 2 & 5 & $2.00446 e+008$ \\
\hline 6 & 2 & 1 & $2.00394 e+008$ \\
\hline
\end{tabular}

6 iterations, total sum of distances $=2.00394 \mathrm{e}+008$ (Pixel or Bit)

في الجدول المرقم (1) يمثل العمود الأول التكرار لخوارزمية العنقدة أما العمود الثاني فيه الطور الذي على أساسه يتم تجزأة البيانات التابعة للصورة المدخلة. العمود الثالث يمثل عدد العناقيد الناتجة في كل تكرار أما العمود الأخير فيعني مجموع المسافات بين العناقيد لكل تكرار. كذلك بالنسبة للجدولين (2) و و(3). 


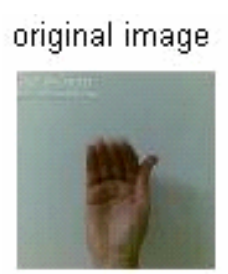

A

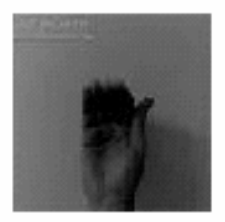

canny filter

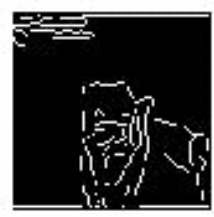

Ksdensity image

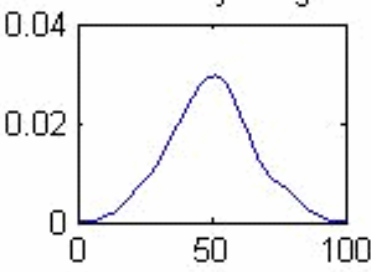

$\mathrm{Y}$

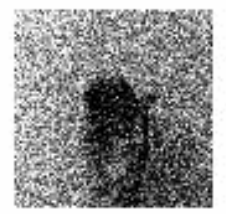

k-means

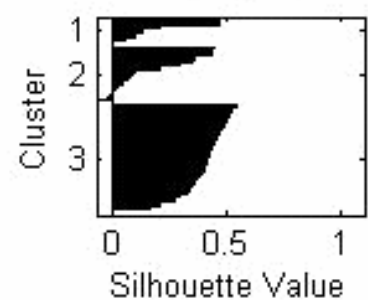

noise image

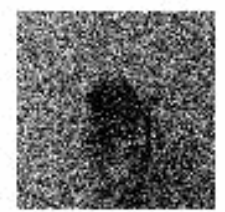

filter noise image

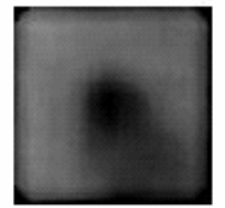

intensity image

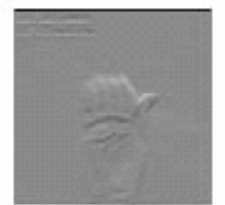

الثكل (2). يمثل الصورة الثانية التي طبقت عليها الخوارزمية اللامعلمية لتقدير دالة صورة إيماءة اليد الاعتيادية مع تقنية

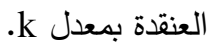

جدول (2). يمثل مراحل تطبيق أسلوب العنقدة بمعدل k-mean) ) لصورة إيماءة اليد الاعتيادية في الثكل (2)

\begin{tabular}{|c|c|c|c|}
\hline iter & phase & num & sum \\
\hline 1 & 1 & 100 & $1.1255 e+007$ \\
\hline 2 & 1 & 13 & $9.6104 e+006$ \\
\hline 3 & 1 & 5 & $9.23469 e+006$ \\
\hline 4 & 1 & 1 & $9.21424 e+006$ \\
\hline 5 & 2 & 0 & $9.21424 e+006$ \\
\hline
\end{tabular}

5 iterations, total sum of distances $=9.21424 \mathrm{e}+006$ (Pixel or Bit)

6. خوارزمية تجزئة وتقدير دالة كثافة الاحتمال لصورة إيماءة اليد المشوهة

Segmentation and Estimator Function Algorithm for Warping Hand Gesture Image

$$
\text { فيما يأتي خطوات الخوارزمية المقترحة: }
$$

Beier-Neely الخطوة الأولى: قراءة الصورة المشوهة الرمادية بعد أخذها من مقطع فيديوي وتطبيق خوارزمية

$$
\text { للتشويه عليها وكالآتي: }
$$

\section{خوارزمية Beier-Neely:}

إن خوارزمية (Beier-Neely) تعتمد على استخدام الأسلوب العكسي من أجل إجراء عملية التشويه

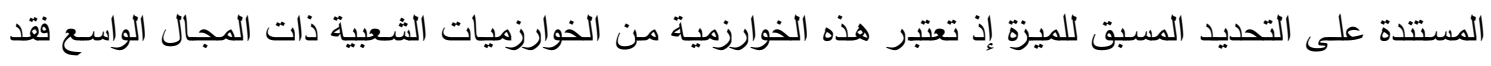
قدمت هذه الخوارزمية من قبل Beier و Neely ونشرت في عام (1992) (والتي تعتبر الميزة أساس التحويل في لعني

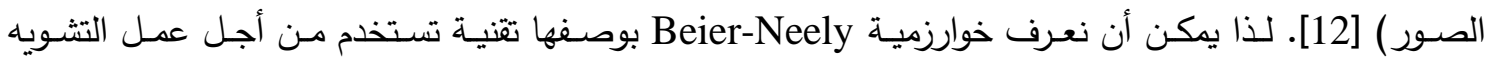


والتركيب للصـور ثنائيسة الأبعـاد لهذا السبب تـعى باسم حقل تركيب الصـور وأن أهم الخطوات الرئيسـة لهـذه الخوارزمية هو العمل على تحديد الميزات للصور المدخلة وتكون على نوعين: خوارزمية Beier-Neely لمتطابقة الخط الوحيد (في حالة الخط الواحد). Single line correspondence Beier-Neely algorithm.

خوارزمية Beier-Neely لمتطابقة الخطوط المتعددة ( في حالة الخطوط المتعددة). Multiple line correspondence Beier-Neely algorithm إن خوارزمية حقل التشويه (في حالة الخط الواحد) يجب إن تحدد فيها بعض الأشياء الضرورية بالنسبة للصورة الدخلة وأيضا للصورة الناتجة. الخطوات من الثانية إلى الثالثة عشر مشابهة تماما لنظيراتها في الخوارزمية السابقة:

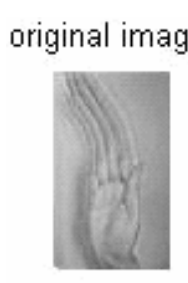

A

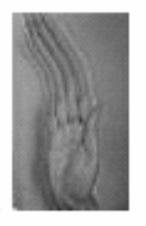

canny filter

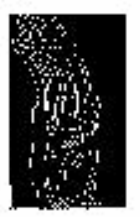

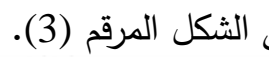
Ksdensity image

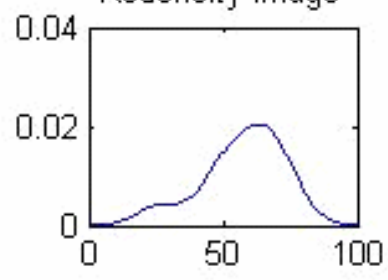

Y

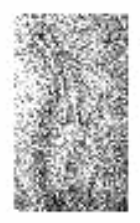

k-means

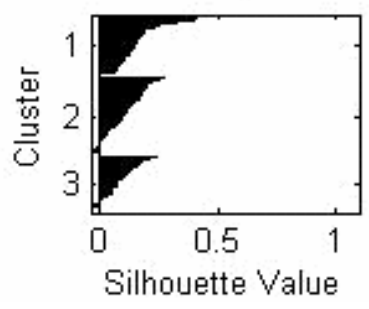

وتطبيق الخوارزمية المقترحة أعلاه ممثل في الثعان noise image

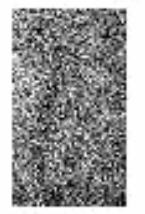

filter noise image

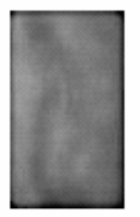

intensity image

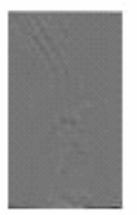

الثكل (3). يمثل الصورة

المشوهة التي طبقت عليها

الخوارزمية اللامعلمية لتقدير دالة كثافة الاحتمال لصورة إيماءة اليد المشوهة مع تقنية العنقدة بمعدل

. $\mathrm{k}$

جدول (3). يمثل مراحل تطبيق أسلوب العنقدة بمعدل k-mean) ) لصورة إيماءة اليد المشوهة في الثكل(3)

\begin{tabular}{|c|c|c|c|}
\hline iter & phase & num & sum \\
\hline 1 & 1 & 100 & $1.20482 e+007$ \\
\hline 2 & 1 & 6 & $1.09183 e+007$ \\
\hline 3 & 1 & 6 & $9.66603 e+006$ \\
\hline 4 & 1 & 3 & $9.24697 e+006$ \\
\hline 5 & 1 & 1 & $9.10073 e+006$ \\
\hline 6 & 1 & 1 & $9.10073 e+006$ \\
\hline 7 & 1 & 1 & $9.03223 e+006$ \\
\hline 8 & 2 & 1 & $9.01808 e+006$ \\
\hline
\end{tabular}

8 iterations, total sum of distances $=9.01808 \mathrm{e}+006$ (Pixel or Bit) 
إن أهم جزء من النتائج التي ظهرت لدينا هو أنه عند استخدام الدالة اللبية (Kernel) على الصورة

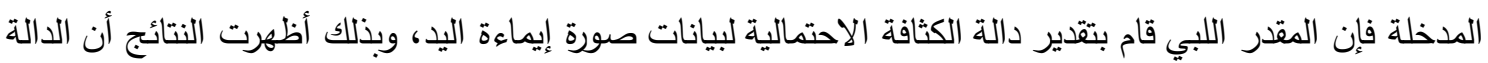

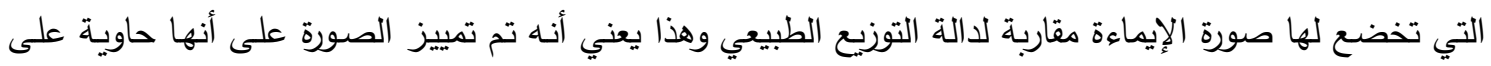

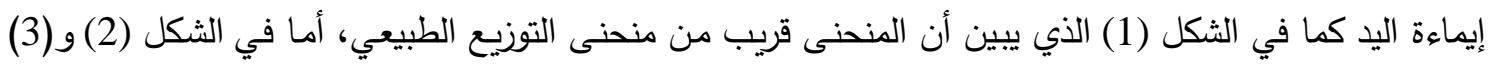
فإن المنحنى يطابق منحنى التوزيع الطبيعي وهذا يعني أنه تم تمييز الصورة على أنها حاوية على صورة إيماءة اليد

وكذلك تم تمييز الصورة المدخلة على أنها صورة حاوية على إيماءة اليد من خلال استخدام تقنية-K) وذلك حسب الثكل الظاهر من خلال الرسم، إذ تم إيضاح أن الرسم ظهر بثلاث مناطق واضحة كعناقيد

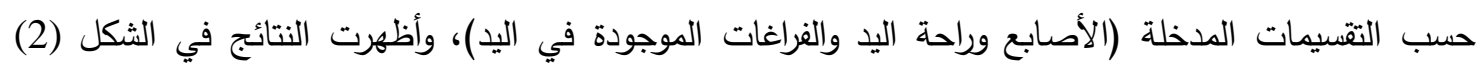
والثكل (3) المناطق الثلاث، أما في الثكل (1) فإن الرسم الظاهر يظهر قيم شاذة لوجود خيال للإيماءة الموجودة

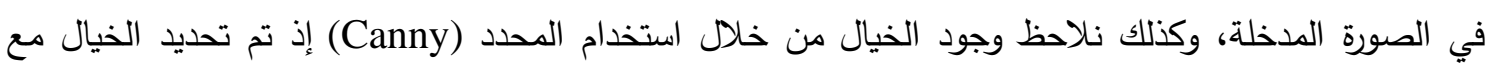
الإيماءة المدخلة.

\section{8. الاستنتاجات Conclusions :}

1. نلاحظ عند استخدام المقدر اللبي Kernel على صورة إيماءة اليد أن المقدر قام بتتعيم بيانات الصورة وتسويتها، وظهر منحني البيانات مشابهاً لمنحني التوزيع الطبيعي، وبذلك تبين لنا أن مقدر الكثافة اللامعلمية

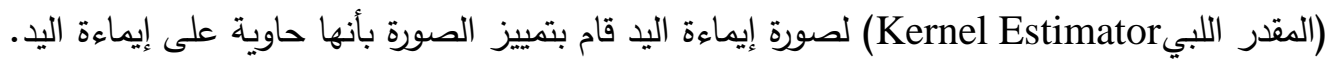
2. يمكن استخدام تقنية (K-Means) لتمييز إيماءة اليد من خلال إدخال تقسيمات إيماءة اليد إلى دالة (K-) لئل .(Means نوصي باستخدام الثبكات العصبية مع نماذج ماركوف المخفية في التعرف على إيماءة اليل. 
|لمصادر

حمـو، منــاف يوسـف، (2000)، "مقارنــة مقدرات Kernel اللامعلميـة لتقدير دوال الانحـدار"، رسـالة

ماجستير غير منشورة، قسم الإحصاء والمعلوماتية، كلية الإدارة والاقتصاد، جامعة بغداد، العراق.

ذنون، باسل يونس، (1997)، "التقدير أللبي" أسلوب بياني في التقدير الإحصائي"، بحث منشور علوم

الرافدين، المجلد 9 ، العدد 1 ، ص صل 89-99.

قاسم، عمر صـابر، (2005)، "استخدام شبكة الانحدار المتدرج المحسنة في تثخيص مرض حصى

الكلى"، رسالة ماجستير غير منشورة، كلية علوم الحاسوب والرياضيات، جامعة الموصل، العراق.

[4] Gasser, T., Muller, H.G. \& Mammitzsch, R. (1985) " Kernels for Nonparametric curve Estimation " J.Royal S.S., series B, Vol.47, No.2, PP238-252.

[5] Hardle, W., (1990): "Applied nonparametric regression", Cambridge, Ma: Cambridge University press.

[6] MATLAB 6.5 The Language of Techical Computing, (2002): "Matlab Help", Math Works Inc.

[7] Parzen, E. (1962), "On estimation of a probability density function and mode“ Ann. Stat. Vol.33, PP1065-1076.

[8] Rabiner, L. R. (1989). "ATutorial on Hidden Markov Models and Selected Applications in Speech Recognition " , Proceeding of IEEE, Vol. 77, No. 2,PP. 257-286.

[9] Schucany, W.R. \& Sommers, J. (1977) " Improved of kernel type density estimators "JASA, Vol.72, No.353, PP.420-423.

[10] Wilson, A.D. and Bobic, A.F.,(1997), "A state Based Approach to the Representation and Recognition of Gesture", Proceedings of IEEE, Vol.19, No.12, PP.1325-1337.

[12] Wolberg, G. (1998), " Image Morphing a Survey ", Dept. of Computer Science New York, NY 10031, No.14. pp 360-375, USA. 